\begin{tabular}{ll|l}
\cline { 2 - 3 } & $\begin{array}{l}\text { Intervent Neurol 2017;6:31-35 } \\
\text { DOI: 10.1159/000452284 } \\
\text { Published online: November 11, 2016 }\end{array}$ & $\begin{array}{l}\text { (c) 2016 S. Karger AG, Basel } \\
\text { www.karger.com/ine }\end{array}$ \\
\hline
\end{tabular}

\title{
Salvage of Herniated Flow Diverters Using Stent and Balloon Anchoring Techniques: A Technical Note
}

\author{
Bharathi D. Jagadeesan ${ }^{a-c} \quad$ Divyajot Sandhu $^{c} \quad$ Kim Jae Hong $^{c}$ \\ Sara Roshan Khanipour ${ }^{a}$ Andrew W. Grande ${ }^{a-c}$ \\ Ramachandra P. Tummala ${ }^{a-c}$ \\ Departments of a Radiology, ${ }^{\mathrm{b}}$ Neurosurgery and ${ }^{\mathrm{C}}$ Neurology, University of Minnesota, \\ Minneapolis, MN, USA
}

\section{Key Words}

Pipeline embolization device $\cdot$ Stent anchor technique $\cdot$ Balloon anchor technique .

Aneurysm

\begin{abstract}
Background: The pipeline embolization device (PED; Medtronic, MN, USA) can sometimes herniate into the aneurysmal sac in an unexpected manner during or shortly after its deployment due to device foreshortening. In this report, we describe 2 endovascular techniques, which can be used to reposition a herniated PED construct into a more favorable alignment. Summary: In a 67-year-old patient who had an intraprocedural herniation of a PED device into a giant cavernous aneurysm, a stent anchor technique was used to reverse the herniation, reorient the PED construct, and achieve successful flow diversion. In a different patient with a giant superior hypophyseal aneurysm, a balloon anchor technique followed by deployment of an LVIS Jr (Microvention, Tustin, CA, USA) stent was used to reverse the herniation into the aneurysmal sac. Key Messages: Stent anchor and balloon anchor techniques as described here can be used to reposition PED constructs, which have unexpectedly herniated into the aneurysm sac during attempted flow diversion for the treatment of giant aneurysms.
\end{abstract}

(C) 2016 S. Karger AG, Base

\section{Introduction}

Flow diverters are increasingly used in the management of large and giant intracranial aneurysms. The pipeline embolization device (PED; Medtronic, MN, USA) remains the only flow diverter, which is FDA approved and widely available in the USA. The PED is a braided 
device and can foreshorten or stretch in vivo, creating challenges in deployment. Occasionally, unfavorable foreshortening of the device with proximal migration of the device can result in herniation of the proximal or distal end of the device into the aneurysm sac [1, 2]. Herein, we describe 2 different techniques, which were used to reposition PED devices following herniation of their distal ends into the aneurysm sac.

\section{Case Reports}

Case 1

A 67-year-old female patient with a wide-necked $1.8 \times 1.4 \mathrm{~cm}$ aneurysm (Fig. 1a, b) arising from the cavernous segment of her left internal carotid artery (ICA) presented with acute onset of painful diplopia. Given the size and location of the aneurysm as well as the wide neck, it was decided to treat this aneurysm by placement of a flow diverter. Under general anesthesia, a Marksman catheter (Medtronic, MN, USA) was advanced into the left ICA through a coaxial system consisting of a 6 F Flexor shuttle guide catheter (Cook Medical, Bloomington, IN, USA) and a 6 French Sofia (Microvention, Tustin, CA, USA) intermediate catheter. Subsequently, a $4.0 \times 25$ mm PED (Pipeline Flex) was advanced through the microcatheter and carefully deployed across the neck of the aneurysm in the usual push and pull fashion, which has been described elsewhere (Fig. 1c). When the microcatheter was then advanced over the delivery wire of the PED in order to recapture the flaps at the end of the delivery wire, the PED unexpectedly foreshortened, and its distal end herniated into the aneurysm. The PED was now positioned such that it was looped within the aneurysm and the distal end faced the proximal ICA (Fig. 1d). The Marksman microcatheter was then advanced through the lumen of the herniated PED, looped inside the aneurysm, and eventually advanced into the distal ICA and proximal middle cerebral artery (MCA). A new $4.5 \times 20 \mathrm{~mm}$ PED was then pushed out of the microcatheter into the proximal MCA so that the distal end of the device could firmly lean against the inner wall of the MCA. Gentle traction was then applied on the Marksman catheter, taking care not to push out more of the device. This resulted in a reduction of the intraaneurysmal loop of the Marksman microcatheter, and the distal end of the previously deployed PED was repositioned into a more favorable position (Fig. 1e). Subsequently, the second PED was gently pulled back from the MCA and deployed within the previous PED in a telescoping fashion (Fig. 1f). The delivery wire of the second PED was then retrieved in a safe manner. Post-PED placement digital subtraction angiography showed stasis of contrast within the aneurysm. At 6 months of follow-up, the patient's visual symptoms had entirely resolved, and the ICA was widely patent with no residual aneurysm filling (Fig. 1g).

Case 2

A 67-year-old female presented with complete painless loss of vision in her right eye. She also had chronic vision loss in her left eye. Subsequently, she was found to have a giant, partially thrombosed superior hypophyseal aneurysm arising from her left ICA. The nonthrombosed part of her aneurysm measured $1.8 \times$ $1.5 \mathrm{~cm}$ and had a 6-mm neck (Fig. 2a). Given the patient's significant medical comorbidities, which excluded surgical management, it was decided to treat the aneurysm with placement of PED, as well as to place a few coils within the aneurysm sac to reduce the chances of post-PED placement aneurysm rupture. A Marksman microcatheter was advanced across the neck of the aneurysm (using the same guide catheter and intermediate catheter system as mentioned with the previous patient), and a Headway 17 catheter was placed within the aneurysm. A $4.5 \times 25 \mathrm{~mm}$ Pipeline Flex device was then successfully deployed across the neck of the aneurysm (Fig. 2b), and 2 coil loops were deployed in the aneurysm; the Headway catheter was then removed. Given the large size of the aneurysm and the ongoing mass effect and vision loss, a second $4.5 \times 25 \mathrm{~mm}$ PED was then placed within the first. However, after placement of the second PED, both PEDs foreshortened such that the distal end of the construct herniated into the aneurysm (Fig. 2c). Multiple attempts were then made to advance a Marksman microcatheter through the lumen of the previously placed PEDs to regain access to the ICA distal to the aneurysm as described above with the previous patient. However, the presence of coil loops within the aneurysm sac prevented looping of the microcatheter and further distal advancement. We then advanced a Scepter XC $4 \times 11 \mathrm{~mm}$ balloon microcatheter through the lumen of the previously placed PED devices, and after looping within the aneurysm sac (around the coil mass), we were able to access the distal ICA. The Scepter XC was then advanced into the M1 segment of the MCA, where it was inflated and gentle traction was applied to straighten the loop within the aneurysm sac and reposition the distal end of the PED construct in a more favorable configuration (Fig. 2d). Subsequently a $2.5 \times 35 \mathrm{~mm}$ LVIS Jr stent was 
Jagadeesan et al.: Salvage of Herniated Flow Diverters Using Stent and Balloon Anchoring Techniques: A Technical Note

Fig. 1. a Frontal projection from a digital subtraction angiogram, showing a wide-necked, large aneurysm arising from the left internal carotid artery in Patient 1. b Lateral projection from a digital subtraction angiogram, showing a wide-necked, large aneurysm. Notice the loop in the large ICA proximal and lateral to the aneurysm. c Oblique image in the same patient. Post-deployment image of the first PED, showing that the PED is properly positioned. d Oblique image following retrieval of the PED delivery wire, showing herniation of the distal end of the PED (white arrow), which now faces backwards to the proximal ICA. e Oblique image showing Marksman microcatheter looped through the aneurysm and advanced to the left MCA with the second PED device within the microcatheter. f Oblique image following deployment of the second PED, showing a new construct with favorable repositioning of the distal end of the first PED. g Follow-up digital subtraction angiogram at 6 months after PED placement in the same patient, showing absence of aneurysm filling. ICA = Internal carotid artery; PED = pipeline embolization device; MCA = middle carotid artery.
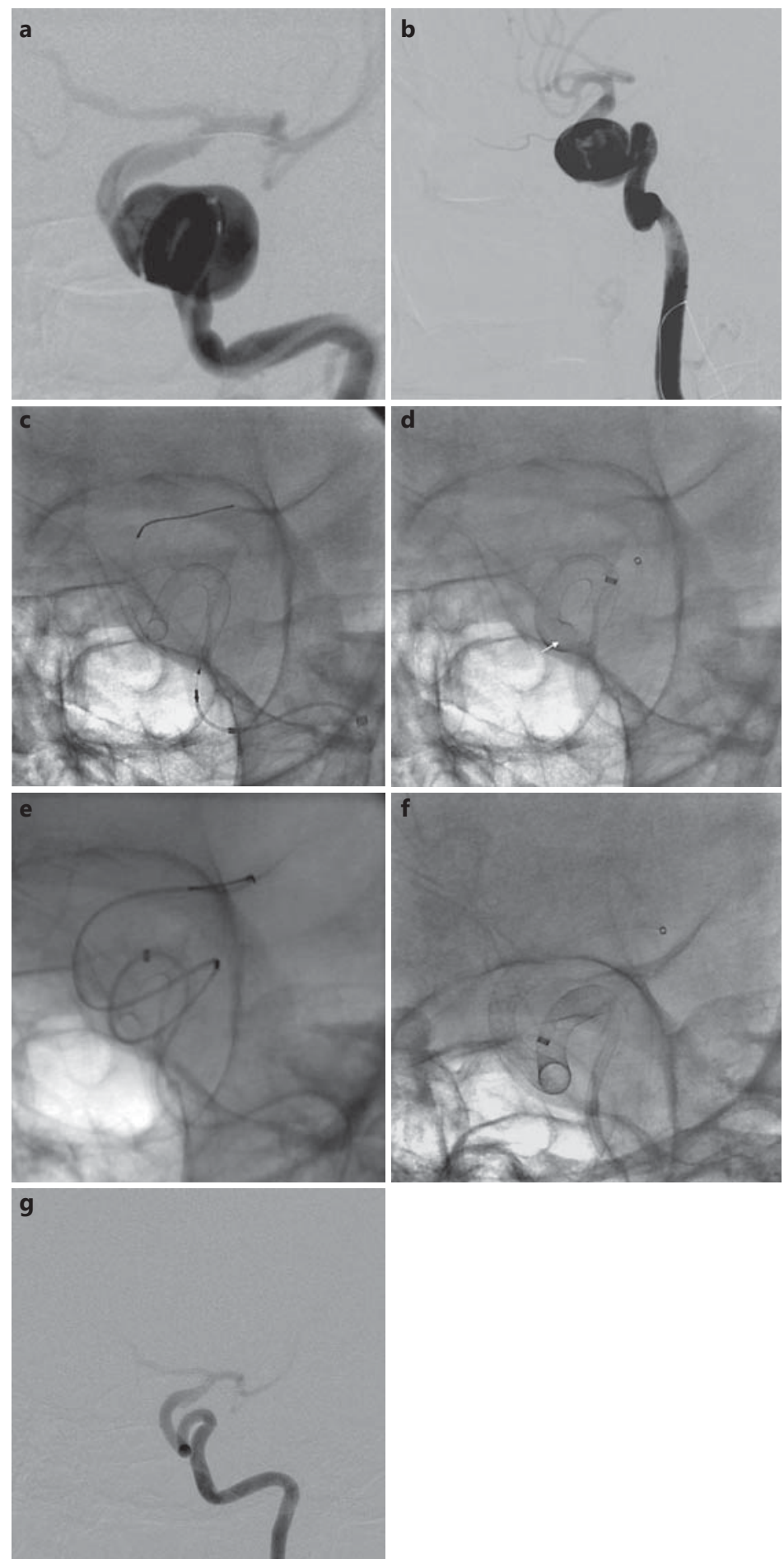

advanced through the Scepter XC balloon microcatheter and deployed such that proximally, it telescoped within the PED construct and distally, it was within the M1 segment of the MCA. This deployment ensured that the PED construct no longer herniated into the aneurysm sac (Fig. 2e). A few more detachable coils were then placed within the aneurysm sac (through a Headway 17 microcatheter, which had been readvanced into the aneurysm sac after navigation of the Scepter XC balloon microcatheter and prior to deployment of the LVIS Jr stent) to significantly reduce the flow within the aneurysm. At 2 months of follow-up, there was a significant improvement in the patient's vision in the right eye. 
Fig. 2. a Frontal projection from a digital subtraction angiogram, showing a partially thrombosed giant left ICA aneurysm in Patient 2. b Magnified frontal projection after deployment of a PED device and coils in the same patient, showing favorable positioning of the PED across the neck of the aneurysm. c Magnified frontal projection in the same patient following placement of the second PED device, showing intra-aneurysmal herniation of the distal end of the construct into the aneurysm (double white arrows). d Magnified frontal view showing a Scepter XC balloon (single white arrow) advanced into the M2 division of the left MCA through the pipeline construct after traction had been applied to the inflated balloon to straighten the intra-aneurysmal loop. e Oblique magnified view showing distal (straight black arrow) and proximal (curved black arrow) ends of an LVIS Jr device placed through the PED construct. ICA = Internal carotid artery; PED = pipeline embolization device; $\mathrm{MCA}=$ middle cerebral artery.

Jagadeesan et al.: Salvage of Herniated Flow Diverters Using Stent and Balloon Anchoring Techniques: A Technical Note
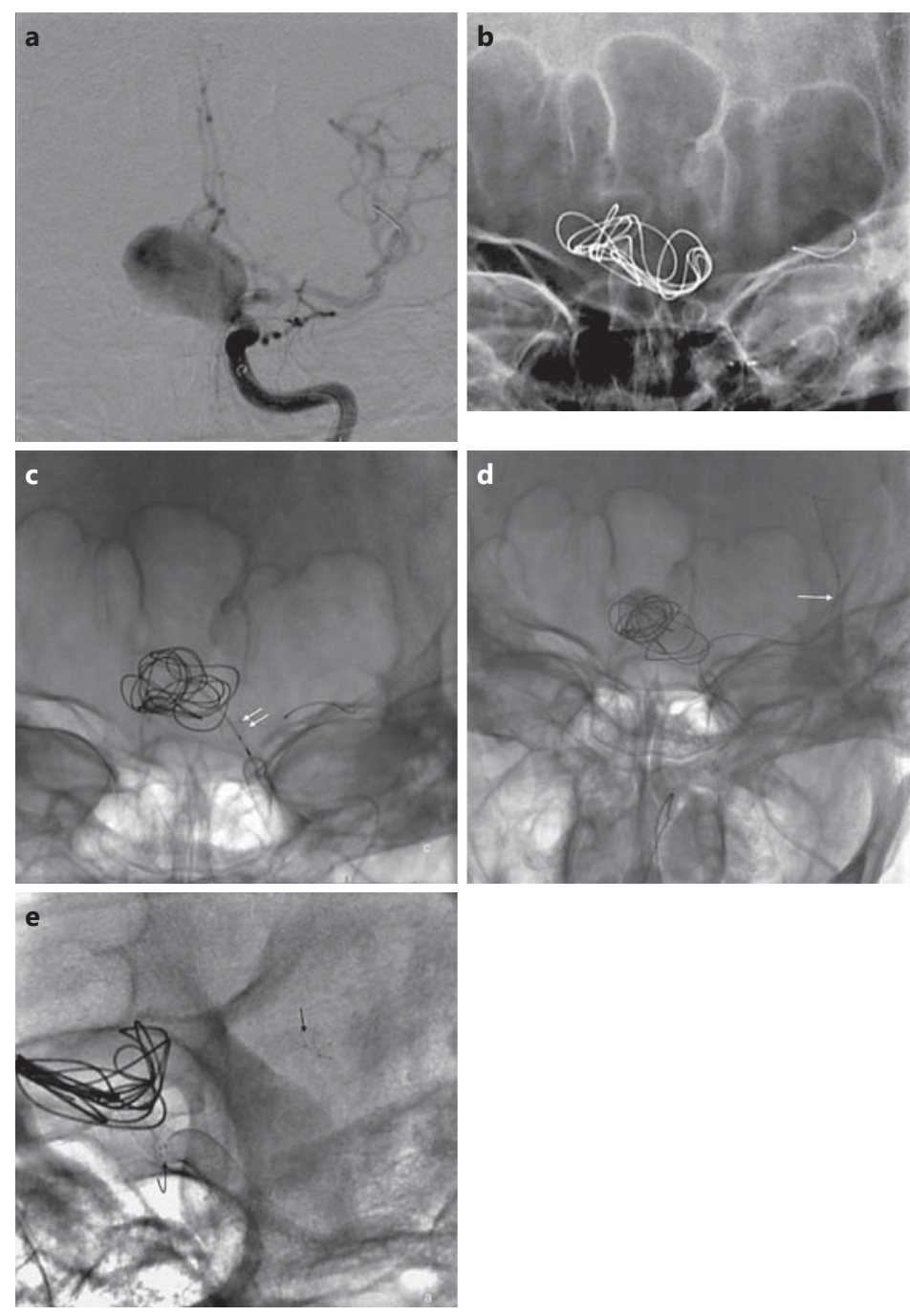

\section{Statement of Ethics}

The Institutional Review Board at the University of Minnesota waives approval for case series involving 2 or less subjects. The subjects' approval was still obtained by the authors.

\section{Discussion}

Herein, we have described our experience with salvage and repositioning of PED constructs following unfavorable foreshortening and intra-aneurysmal herniation of the distal end of the constructs. In 1 patient, we used a balloon anchor technique, and in the other, a PED anchor technique. The balloon anchor technique has been previously described for navigation across wide-necked giant aneurysms [3-5]. The stent anchor technique has been used for reduction of intra-aneurysmal loops during PED deployment prior to device placement [6]. However, as we have shown, these techniques can also be used to salvage and reposition unfavorable intra-aneurysmal herniation of PED constructs. A similar technique wherein a balloon microcatheter was used in addition to a device delivery catheter has been described previously [7]. In that report, the authors used a single lumen balloon to reorient the PED and then advanced a second PED delivery microcatheter alongside into the distal 
vessel. However, in our second patient, we chose to use a dual lumen balloon and deploy a stent directly within the pipeline construct. Familiarity with the techniques described here as well as those described by earlier authors can help practitioners overcome challenges posed by unexpected intra-aneurysmal herniation of PED constructs. Additionally, it must be emphasized that, when possible, landing the PED devices quite distal to the aneurysm neck to begin with can also minimize the incidence of foreshortening and intra-aneurysmal herniation.

\section{Conclusion}

It is possible to salvage unanticipated intra-aneurysmal herniation of the distal ends of flow diverters by resorting to a balloon anchor or stent anchor technique.

\section{Disclosure Statement}

Dr. Jagadeesan is a consultant for Medtronic, the manufacturers of PED, and Microvention, the manufacturer of the LVIS Jr stent and Scepter XC balloon.

Dr. Grande is a consultant for Medtronic. The other authors have no conflicts of interest to disclose.

\section{References}

1 Mitchell B, Jou L-D, Mawad M: Retrieval of distorted pipeline embolic device using snare-loop. J Vasc Interv Neurol 2014;7:1-4.

2 Hauck EF, Natarajan SK, Langer DJ, Hopkins LN, Siddiqui AH, Levy EI: Retrograde trans-posterior communicating artery snare-assisted rescue of lost access to a foreshortened pipeline embolization device: complication management. Neurosurgery 2010;67:495-502.

3 Ding D, Starke RM, Evans AJ, Jensen ME, Liu KC: Balloon anchor technique for pipeline embolization device deployment across the neck of a giant intracranial aneurysm. J Cerebrovasc Endovasc Neurosurg 2014;16: 125-130.

4 Peeling L, Fiorella D: Balloon-assisted guide catheter positioning to overcome extreme cervical carotid tortuosity: technique and case experience. J Neurointerv Surg 2014;6:129-133.

5 Martínez-Galdámez M, Ortega-Quintanilla J, Hermosín A, Crespo-Vallejo E, Ailagas JJ, Pérez S: Novel balloon application for rescue and realignment of a proximal end migrated pipeline flex embolization device into the aneurysmal sac: complication management. BMJ Case Rep 2016;2016:bcr2016012263.

6 Clarençon F, Wyse G, Fanning N, Di Maria F, Gaston A, Chiras J, et al: Solitaire FR stent as an adjunctive tool for pipeline stent deployment in the treatment of giant intracranial aneurysms. Neurosurgery 2013;72(2 suppl operative):onsE241-E244; discussion onsE244.

7 Crowley RW, Abla AA, Ducruet AF, McDougall CG, Albuquerque FC: Novel application of a balloon-anchoring technique for the realignment of a prolapsed pipeline embolization device: a technical report. J Neurointerv Surg 2014;6:439-444. 